\title{
COMMENTARY
}

\section{Measuring glomerular filtration rate in acute kidney injury: Yes, but not yet}

\author{
Bruce A Molitoris* \\ See related research by Pickering et al., http://ccforum.com/content/16/3/R107
}

\begin{abstract}
Acute kidney injury has become a major focus for nephrologists and critical care physicians. The development of structural biomarkers is proceeding, but the results to date have been disappointing. The use of a shortened creatinine clearance as a functional acute kidney injury biomarker is not new but has not been compared with that of other diagnostic approaches. A rapid, repeatable, and accurate measured glomerular filtration rate would be the gold standard for a functional biomarker and is not far off.
\end{abstract}

Acute kidney injury (AKI) remains a vexing clinical problem resulting in unacceptably high patient mortality, development of chronic kidney disease, and enhanced progression to end-stage kidney disease [1]. Although clinical risk factors for developing AKI have been identified, there is no reasonable surveillance technique ('biomarker') to either definitively and rapidly diagnose the injury or determine the extent of its severity. Since patient outcomes correlate with the extent of injury and effective therapy requires early intervention, the ability to rapidly diagnose and stratify patients by their level of kidney injury is of paramount importance for therapeutic progress. Therefore, a search for a biomarker of kidney injury has intensified and is now considered by many experts to be the highest priority in the field of AKI [2]. It is likely that a combination of structural and functional markers of AKI will provide the highest clinical utility.

Glomerular filtration rate (GFR), which measures the amount of plasma filtered through glomeruli within a given period of time, is clinically the most widely used indicator of kidney function. Yet a rapid quantitative

*Correspondence: bmolitor@iupui.edu

Department of Medicine, Division of Nephrology, Indiana University School of Medicine, Research Building \#2, RM202, 950 West Walnut Street, Indianapolis, IN 46202, USA technique with clinical utility has not been developed. Reduction in the GFR, secondary to kidney injury, is the hallmark of AKI and results in increased levels of blood urea nitrogen (BUN) and serum creatinine. Unfortunately, the rates of increase in BUN and serum creatinine do not parallel the fall in GFR in a time frame that is clinically useful. In addition, since both creatinine production from muscle and GFR determine the serum creatinine level, using serum creatinine as an indicator of GFR is highly patient-specific and often problematic or even misleading. These issues have been described elsewhere [3].

Achieving the ability to rapidly and accurately measure GFR in an early stage of AKI would be beneficial for many reasons. It would rapidly identify and determine the extent of injury, allowing early pharmacologic or dialytic treatment (or both), enrollment and stratification for clinical studies, and prognostic information. It could also be used to determine the effect of a clinical maneuver on GFR, such as volume resuscitation and the use of pressors to support blood pressure, and enable appropriate drug dosing for agents cleared by the kidney or nephrotoxins.

On this well-known background, Pickering and colleagues [1] set out to determine the clinical utility of a 4-hour creatinine clearance $(\mathrm{CrCl})$, compared with plasma creatinine, for diagnosing AKI. The authors found that $\mathrm{CrCl}$ increased the likelihood of diagnosing AKI; a decreasing $\mathrm{CrCl}$ correlated with increased kidney injury severity, death, or dialysis; and the $\mathrm{CrCl}$ was most helpful when patients began with a serum creatinine in the normal range. Although the receiver operating characteristics were not impressive, the study was a small pilot study.

This is not the first time a shortened $\mathrm{CrCl}$ has been used to estimate GFR in clinical settings. HergetRosenthal and colleagues [4] used a 2-hour $\mathrm{CrCl}$ in stable patients and found an acceptable and repeatable correlation with the 24-hour $\mathrm{CrCl}$. However, the accuracy and utility of a shortened collection in unstable patients were questioned by two studies conducted in patients with AKI $[5,6]$. This limitation may relate to reduced production of creatinine in sepsis [7], increased production 
of creatinine with trauma, increased metabolism including the use of glucocorticoids, or the changing of GFRs during the collection periods. Pickering and colleagues did not attempt to validate their 4-hour $\mathrm{CrCl}$ rates with 6-hour iohexol or iothalamate infustion studies, currently the gold standard for clinical studies. This validation could have been done nearly simultaneously with the 4-hour $\mathrm{CrCl}$ urine collection. Until such studies are conducted, confidence for using a $\mathrm{CrCl}$ may be limited.

A clinically useful technique to measure GFR in AKI has been a long-sought-after goal [8]. The cumbersome, time-consuming, and expensive techniques currently available have not met this important clinical need. The development and use of estimating formulas based on serum creatinine or cystatin $\mathrm{C}$ have been disappointing for several well-explained reasons [3]. Therefore, the development of a rapid, accurate, safe, easy, and inexpensive technique has high clinical importance both in and out of the hospital. An approach being developed commercially, used primarily in preclinical studies to date, is the use of bedside techniques to measure GFR. The use of inexpensive, nontoxic, easily sized fluorescent molecules has emerged as a likely candidate, and several groups are now working with this approach in preclinical models $[9,10]$. It is likely that several of these approaches will enter clinical phase studies, and improvements in design and clinical utility are continually being developed. Test rapidity, convenience, cost, safety, accuracy, and repeatability are all critical characteristics. Hopefully, one or more of these approaches will emerge and alleviate the need for multiple-hour urine collection and analysis.

Abbreviations

AKI, acute kidney injury; BUN, blood urea nitrogen; $\mathrm{CrCl}$, creatinine clearance; GFR, glomerular filtration rate.

\section{Competing interests}

BAM is a co-founder and part owner and the medical director of FAST BioMedical (Indianapolis, IN, USA)

Published: 24 September 2012

\section{References}

1. Pickering JW, Frampton CM, Walker RJ, Shaw GM, Endre ZH: Four hour creatinine clearance is better than plasma creatinine for monitoring renal function in critically ill patients. Crit Care 2012, 16:R107.

2. Hewitt SM, Dear J, Star RA: Discovery of protein biomarkers for renal diseases. J Am Soc Nephrol 2004, 15:1677-1689.

3. Pisitkun T, Shen RF, Knepper MA: Identification and proteomic profiling of exosomes in human urine. Proc Natl Acad Sci U S A 2004, 101:13368-13373.

4. Herget-Rosenthal S, Kribben A, Pietruck F, Ross B, Philipp T: Two by two hour creatinine clearance--repeatable and valid. Clin Nephrol 1999, 51:348-354.

5. Cherry RA, Eachempati SR, Hydo L, Barie PS: Accuracy of short-duration creatinine clearance determinations in predicting 24-hour creatinine clearance in critically ill and injured patients. J Trauma 2002, 53:267-271.

6. Herrera-Gutiérrez ME, Seller-Pérez G, Banderas-Bravo E, Muñoz-Bono J, Lebrón-Gallardo M, Fernandez-Ortega JF: Replacement of 24-h creatinine clearance by 2 -h creatinine clearance in intensive care unit patients: a single-center study. Intensive Care Med 2007, 33:1900-1906.

7. Doi K, Yuen PS, Eisner C, Hu X, Leelahavanichkul A, Schnermann J, Star RA: Reduced production of creatinine limits its use as marker of kidney injury in sepsis. J Am Soc Nephrol 2009, 20:1217-1221.

8. Dagher PC, Herget-Rosenthal S, Ruehm SG, Jo SK, Star RA, Agarwal R, Molitoris BA: Newly developed techniques to study and diagnose acute renal failure. J Am Soc Nephrol 2003, 14:2188-2198.

9. Poreddy AR, Neumann WL, Freskos JN, Rajagopalan R, Asmelash B, Gaston KR, Fitch RM, Galen KP, Shieh JJ, Dorshow RB: Exogenous fluorescent tracer agents based on pegylated pyrazine dyes for real-time point-of-care measurement of glomerular filtration rate. Bioorg Med Chem 2012 20:2490-2497.

10. Wang E, Meier DJ, Sandoval RM, Von Hendy-Willson VE, Pressler BM, Bunch RM, Alloosh M, Sturek MS, Schwartz GJ, Molitoris BA: A portable fiberoptic ratiometric fluorescence analyzer provides rapid point-of-care determination of glomerular filtration rate in large animals. Kidney Int 2012, 81:112-117.

\section{doi:10.1186/cc11482}

Cite this article as: Molitoris BA: Measuring glomerular filtration rate in acute kidney injury: Yes, but not yet. Critical Care 2012, 16:158. 